\title{
Pain management knowledge among medical ward nurses in Malaysia
}

\begin{abstract}
Pain, a common symptom for patients in medical wards, can be relieved by effective pain management, with nurses playing a vital role in this regard. A study was undertaken to determine the knowledge of nurses on pain management and to examine the factors that influence their acquisition of such knowledge. A total of 143 medical ward nurses (representing 60\% response) in a government referral hospital in Malaysia participated in the study. Data from this study derived from a set of questionnaires were analysed using $2 \times 2$ contingency tables and the chi squared test with Yateôs correction. Approximately two thirds of the nurses were deficit in knowledge regarding pain management, registering a mean knowledge score of 40.5 out of a possible 100 points. There was no significant association between the level of knowledge on pain management and demographic characteristics such as age and work experience. There was also no significant relationship between the level of knowledge on pain management and the pain courses attended. A better understanding of the factors that affect the acquisition of such knowledge could provide useful information that can be incorporated in an improved educational program on pain management for nurses.
\end{abstract}

Keyword: Pain management; Medical wards; Nurses; Malaysia 